\title{
CSF Corticotropin-Releasing Factor in Personality Disorder: Relationship with Self-Reported Parental Care
}

\author{
Royce J Lee*,', Jackie Gollan', John Kasckow', Thomas Geracioti' and Emil F Coccaro' \\ 'Clinical Neuroscience \& Psychopharmacology Research Unit, Department of Psychiatry, The University of Chicago, Chicago, IL, USA
}

\begin{abstract}
This cross-sectional investigation tests the relationship between the level of self-reported childhood parental care and cerebrospinal fluid (CSF) corticotropin-releasing factor (CRF) concentration in adults with and without personality disorder (PD). Based on preclinical models of the lasting effect of post-natal parental care on central CRF function, the primary hypothesis was that childhood parental care, as reflected by the parental bonding inventory (PBI) care and involvement subscale, is inversely correlated with adult CSF CRF levels. The sample includes cerebrospinal fluid CRF samples from 19 subjects who were included in a previously published report on the relationship between CRF level and Childhood Trauma Questionnaire score. Parental bonding was measured retrospectively with the PBI in 54 medication-free male and female subjects, 37 of whom were diagnosed with a DSM-IV PD, 17 of whom were normal controls free of Axis I and II psychopathology. CSF CRF level as measured by lumbar puncture was entered into a model as the dependent variable, with the independent variables of PBI Parental Care and Involvement, diagnostic category, age, and gender. The model predicting CSF CRF level was significant, with PBI parental care and involvement negatively correlated with CSF CRF level. PD subjects with higher than median PBI parental care and involvement score had significantly lower CSF CRF levels than both normal controls and PD subjects with lower than median PBI parental care and involvement. Higher levels of self-report parental care predict lower CSF CRF levels in PD subjects, consistent with a beneficial effect of parental care on decreased stress reactivity, and consistent with previous reports in humans. The cross-sectional design of the study, however, limits causal inferences.

Neuropsychopharmacology (2006) 31, 2289-2295. doi: I 0. I038/sj.npp. I 30I I04; published online 26 July 2006
\end{abstract}

Keywords: corticotropin releasing factor; corticotrophin releasing hormone; child abuse; childhood trauma; personality disorder; borderline personality disorder

\section{BACKGROUND}

The development of personality disorder (PD) has been postulated to arise from interacting genetic and environmental determinants (Goodman et al, 2004), although specific genetic risk factors are still being studied. Consistent with cross-sectional studies reporting associations between PD and childhood abuse and/or neglect (Brierer et al, 2003), documented parental abuse and neglect have been longitudinally linked to the development of PD in early adulthood (Johnson et al, 1999). In the absence of controlled, experimental proof, these data provide indirect support for a causal role for childhood abuse and/or neglect in the development of PD. It is also possible that childhood abuse and/or neglect modify the course or severity of PD, as

\footnotetext{
* Correspondence: Dr RJ Lee, Clinical Neuroscience \& Psychopharmacology Research Unit, Department of Psychiatry, The University of Chicago, 584I South Maryland Avenue, Chicago, IL 60637, USA, Tel: + I 773834 5673, Fax: + I 773834 4536,

E-mail: rlee@yoda.bsd.uchicago.edu

Received 6 December 2005; revised 7 March 2006; accepted 27 March 2006

Online publication: 18 April 2006 at http://www.acnp.org/citations/ Npp04| 806050723/default.pdf
}

has been found to be the case for other psychiatric disorders such as bipolar disorder (Garno et al, 2005). The biological mechanism whereby childhood abuse and/or neglect may increase the risk for personality psychopathology is not understood.

Preclinical models of the effect of childhood parental care have found that disruptions in parental care, especially in the post-natal, pre-weaning period, result in persistent elevations in central CRF drive (Coplan et al, 1998; Nemeroff, 2004). Convergent human data is available from two published cross-sectional reports of a positive relationship between cerebrospinal fluid (CSF) level of corticotropin-releasing factor (CRF) and history of childhood abuse and/or neglect. The first found that in depressed and normal control subjects, cerebrospinal fluid (CSF) level of CSF CRF, but not depressive disorder, was positively related to level of early childhood parental abuse and/or neglect as measured by a questionnaire measure of perceived early life stress (Global perceived early life stress; Carpenter et al, 2004). We subsequently reported that in a sample of 20 male PD subjects, CSF CRF was positively correlated with Childhood Trauma Questionnaire total and the Emotional Neglect subscale score (Lee et al, 2005). The behavioral consequence of persistent central CRF elevations in humans 
is unknown. Because of the important role of brain CRF in neural circuits underlying emotional and social behaviors, elevations in CRF may have a role in mood, anxiety, and PDs.

Parental care is the conceptual opposite of childhood abuse and/or neglect. We chose to measure parental care in childhood with the widely used Parental Bonding Inventory (PBI; Parker et al, 1979), a self-report questionnaire with demonstrated reliability that asks subjects to recall patterns of parental behaviors such as emotional warmth and overprotectiveness. In this study, we were specifically interested in the PBI Parental Care and Involvement subscale and its relationship to adult levels of CSF CRF. We hypothesized that higher levels of experienced parental care would predict lower CSF CRF levels in adulthood. Additionally, the following categorical hypothesis was tested: that PD subjects reporting less childhood parental care would have higher CSF CRF levels than PD subjects reporting more childhood parental care and normal controls. This sample included the 19 subjects on whom was previously reported Childhood Trauma Questionnaire (a different measure of early life environment inversely related to parental care) and CSF CRF data (Lee et al, 2005). This sample also included additional male and female subjects, and a non-PD normal control group.

\section{SUBJECTS AND METHODS}

\section{Subjects}

Informed, written consent was obtained for the 56 consecutively recruited, physically healthy subjects with complete CSF monoamine/neuropeptide and PBI Parental Care data. The IRB-approved protocol included diagnostic and questionnaire assessments, and lumbar puncture, as part of a larger program designed to study the biological correlates of personality. Thirty-nine PD and 17 non-PD subjects were included in the analysis. Data from 19 of the male subjects in this sample were previously presented in a study of the relationship between Childhood Trauma Questionnaire score and level of CSF CRF (Lee et al, 2005). The subjects were recruited by newspaper and public service announcements seeking subjects with and without self-reported problems associated with PD (ie relationship problems, mood problems, impulsivity). Subjects were excluded from study for history of bipolar I and II disorder, cyclothymia, any history of psychotic disorder other than micropsychotic episodes related to Axis II psychopathology, and current alcohol or substance dependence (see below). Given the high rate of comorbidity between major depressive disorder (MDD) and PD, we did not exclude subjects with current MDD but assessed the effect of current MDD on results of analyses. Subjects were medically excluded for active medical problems detected by medical history, significantly abnormal laboratory values (including complete blood count with platelets and differential, serum electrolytes, blood urea nitrogen and creatinine, liver function, thyroid stimulating hormone (TSH), urinary analysis and toxicology), significantly abnormal electrocardiogram, history of bleeding abnormalities, or abnormal physical exam. Also excluded from study were females with positive serum $\beta$-HCG (pregnancy) test. None of the subjects were taking prescribed psychotropic medication at time of study. Moreover, all subjects were instructed to remain free of all drugs for at least 2 weeks before study and to follow a low monoamine diet, which proscribes ingestion of foods with known high monoamine content such as fermented beverages and smoked meats (Cutler and Hodes, 1983), for at least 3 days before study.

\section{Diagnostic Assessment}

DSM-IV Axis I and II diagnoses were established using information from (a) semi-structured interviews by trained clinicians following the Schedule for Affective Disorders and Schizophrenia (Spitzer et al, 1978) for Axis I disorders and the Structured Interview for the Diagnosis of DSM PD (Pfohl et al, 1989), updated for DSM-IV Axis II disorders, (b) clinical interview by a research psychiatrist, and (c) review of all other available clinical data. Diagnosis of alcoholism was made by modified Research Diagnostic Criteria (RDC) (Coccaro et al, 1996). Information was reviewed and diagnoses established by best-estimate procedures previously described (Coccaro et al, 1996). The best-estimate procedure allows data gathered during semi-structured interviews by clinical raters to be reviewed by psychiatrists with clinical experience with difficult to diagnose conditions such as bipolar II and cyclothymic disorder. Subject diagnostic information is displayed in Tables 1 and 2.

\section{Measurement of Self-Reported Parental Care}

Self-reported parental care, the subjective perception and recollection of quality of parental care received in child-

Table I Subject Characteristics $(n=54)$

\begin{tabular}{lcc}
\hline & PD (n=37) & Normal control $(\mathbf{n}=\mathbf{1 4})$ \\
\hline Gender $(\%(n))$ & - & - \\
Male & $75.7 \%(28)$ & $82.4 \%(14)$ \\
Female & $24.3 \%(9)$ & $17.6 \%(3)$ \\
& & - \\
Race (\%(n)) & - & $52.9 \%(9)$ \\
Caucasian & $70.3 \%(26)$ & $23.5 \%(4)$ \\
African -American & $29.7 \%(11)$ & $5.9 \%(1)$ \\
Asian & $0 \%(0)$ & $11.8 \%(2)$ \\
Hispanic & $0 \%(0)$ & $5.9 \%(1)$ \\
Asian Indian & $0 \%(0)$ & $174.2 \pm 8.3$ \\
& & $74.9 \pm 11.1$ \\
Height (mean cm $\pm S D)$ & $173.7 \pm 13.7$ & $30.7 \pm 8.6$ \\
Weight (mean kg $\pm S D)$ & $72.7 \pm 13.6$ & $84.5 \pm 3.8 *$ \\
Age (mean years $\pm S D)$ & $32.4 \pm 7.7$ & $0 \%(0)$ \\
GAF (mean $\pm S D)$ & $58.2 \pm 10.7 *$ &
\end{tabular}

$*=p<0.05$, indicating significant difference between PD and normal group. Demographic information on the entire sample, PD subgroup, and non-PD normal subgroup. As expected, PD subjects had significantly lower GAF score compared to normal subjects. The PD subgroup had clinically significant levels of psychopathology, as indicated by the $21.6 \%$ suicide attempt rate. 
Table 2 Axis I and Axis II Disorders in the PD Group $(n=37)$

\begin{tabular}{lr}
\hline Diagnosis & \% (n) \\
\hline Paranoid & $21.6(8)$ \\
Schizoid & $5.4(2)$ \\
Schizotypal & $0(0)$ \\
Antisocial & $13.5(5)$ \\
Borderline & $16.2(6)$ \\
Histrionic & $8.1(3)$ \\
Narcissistic & $8.1(3)$ \\
Avoidant & $5.4(2)$ \\
Dependent & $2.7(1)$ \\
Obsessive-compulsive & $13.5(5)$ \\
PD-NOS & $40.5(15)$ \\
Major depression & $5.4(2)$ \\
Dysthymia & $8.1(3)$ \\
PTSD & $0(0)$ \\
GAD & $0(0)$ \\
\hline
\end{tabular}

hood, was measured by the parental bonding inventory (PBI; Parker et al, 1979) Parental Care subscale score. The PBI is a 25-item self-rating scale, with each item measuring on a 4-point Likert scale qualitative aspects of individual parent (mother and father) behavior. The PBI parental care and involvement (PBI Care) subscale score represents a summation of data regarding perceived parental care from the mother and father from one of two dimensions of parental care (care $v s$ neglect). The PBI Care score shows good test-test reliability $(0.761)$ as well as excellent correspondence with results of an interview assessment of parental attachment. PBI Care describes experienced affection, emotional warmth, empathy and closeness vs emotional coldness, indifference, and neglect. PBI Care has no association with age, and only weak associations with social class. The second PBI dimension, Overcontrol, was not analyzed due its lower reliability and uncertain validity (Parker et al, 1979). In 35 of the subjects who completed both the PBI and the childhood trauma questionnaire (CTQ: Bernstein and Fink, 1988), PBI parental care and CTQ total score were significantly negatively correlated $(r=-0.852$, $p<0.01, n=35$ ). The strong, significant negative correlation indicates that low scores on PBI Care predict greater perceived history of childhood abuse and/or neglect.

\section{Lumbar Puncture}

Subjects reported to the Clinical Procedures Lab at approximately 2100 hours the evening before the Lumbar Puncture procedure. After admission, subjects rested in a quiet, comfortable room with a television. No medical or experimental procedures were conducted. The next morning, approximately $1 \mathrm{~h}$ before lumbar puncture was performed, subjects completed a visual analogue scale (VAS) of subjective mood state. At 1000 hours, lumbar punctures were performed under sterile technique by a research neurologist, using intradermal lidocaine for local anesthesia. A total of $20 \mathrm{cc}$ of CSF was withdrawn in six aliquots: aliquots $1,2,4,5$ and 6 each consisted of $1 \mathrm{cc}$ of CSF and were set aside for future analyses. Aliquot 3 was composed of one pooled $15 \mathrm{cc}$ sample of CSF, subsequently subdivided into $151 \mathrm{cc}$ subaliquots for later analysis. One of the identical sub-aliquots from Aliquot 3 was used for assay of CSF CRF. All CSF samples were frozen immediately at $-70^{\circ} \mathrm{C}$ until assay. Assay of CRF and adrenocorticotropin hormone (ACTH) were by radioimmunoassay (RIA) using reagents provided by IgG Corporation (Nashville, TN). CSF $\mathrm{CRF}$ inter- and intra-assay coefficients of variability were 11.9 and $7.2 \%$, respectively (Kasckow et al, 2001).

\section{Statistical Analysis}

CSF CRF concentration and PBI Care and Involvement score (PBI Care) were checked for normality of distribution and outliers by inspection of histogram plots and onesample Kolmogorov-Smirnov tests. Demographic and health-related variables were tested as potential confounds of the relationship between CSF CRF and PBI Care using Pearson's correlation for continuous variables (age, weight, height) and t-tests for dichotomous variables (gender, smoking history). The primary dimensional hypothesis of the study was tested utilizing linear regression analysis, predicting variance in CSF CRF with a model that included in the first step age and gender (male $=1 v s$ female $=2)$, in the second step diagnostic category $(\mathrm{PD}=1$ vs $\mathrm{NC}=2)$ and PBI Care, and in the third step the interaction between the $z$-score of PBI Care and diagnostic category (PBI Care $z$-score $\times$ diagnostic category). To follow up on the potential interaction effect (PBI Care -score $\times$ diagnostic category), Pearson's correlation coefficients between CSF CRF and PBI Care were calculated separately in the normal control and PD groups, using the Fischer's $Z$ transformation. Exploratory analyses were conducted within the PD group excluding the two currently depressed subjects, as depression may be etiologically related to both early environment and CSF CRH level. Next, to test if the relationship between CSF CRF and PBI Care was driven by the 19 subjects presented in our previous study (Lee et al, 2005), Pearson's correlation was conducted between CSF CRF and PBI Care in the new set of subjects, excluding the 19 original subjects and two depressed subjects.

To test the secondary categorical hypothesis, that PD subjects who report less childhood parental care (Low PBI PD) have higher CRF levels than both normal controls and PD subjects reporting more childhood parental care (High PBI PD), subgroup differences in CSF CRF levels were tested using ANOVA for CSF CRF level, with the between-group factor of subject group (normal control $v s$ High PBI PD vs Low PBI PD). Finally, exploratory comparisons of CSF CRF between specific PD categories and normal control subjects were performed where adequate sample sizes were available.

\section{RESULTS}

PD subjects compared to non-PD healthy subjects had significantly lower levels of global psychosocial function as measured by Global Assessment of Function (GAF) score $(85 \pm 9$ vs $58 \pm 11, \mathrm{t}(54)=9.99, p<0.01)$ but did not differ 
in height, weight, BMI, and age. Neither gender nor recent smoking history predicted significant differences in CSF CRF and PBI Care. Two PD subjects had CSF CRF levels greater than 3 standard deviations above the mean (141.9 and $106.5 \mathrm{pg} / \mathrm{ml}$ ); given that these values were greater than 3 standard deviations above the mean of previously reported studies in psychiatric populations, data from these subjects was excluded (see Discussion). After exclusion of these two outliers, CSF CRF had a normal distribution by K-S test in the entire sample $(n=54)$ and in the PD $(n=37)$ and normal control groups $(n=17)$ separately. PD subjects do not have a statistically higher mean level of CRF level compared to normal controls $(35.497 \pm 19.8$ vs $27.135 \pm 13.233 \mathrm{pg} / \mathrm{ml}$ ) but had a significantly lower mean PBI Care score and a greater range of scores $(19.7 \pm 9.5$, range $=1-36$, vs $24.8 \pm 5.8$, range $=15-32$ ). None of the control subjects endorsed very low levels of parental care and involvement, while $30 \%$ of the PD subjects endorsed very low levels of parental care and involvement, with PBI Care $<15$.

The model predicting CSF CRF level, after accounting for age and sex, predicted $18 \%$ of variance in CSF CRF (see Table 3), with PBI Care independently, negatively predicting CSF CRF level while diagnostic category did not predict CSF CRF level. Addition of the interaction term ( $z$-score PBI Care $\times$ diagnostic category) resulted in a stronger model accounting for $24 \%$ of variance in CSF CRF, with the interaction term predicting CSF CRF level at a trend level of significance. The probe of the interactive effect of diagnostic group on the relationship between CSF CRF and PBI Care revealed that the correlation between CSF CRF and PBI Care was significant in the PD group $(r=-0.401, p=0.014$, $n=37)$ but not the normal control group $(r=0.297$, $p=0.247, n=17)$. The correlation coefficients in the two groups were significantly different, using Fischer $Z$ transformation ( $Z$ diff $=2.302, p=0.021)$. It is important to note that the range of PBI scores was restricted in the normal control group, in which no subject endorsed very low levels of parental care.

The results of exploratory analyses conducted within the PD group are as follows. Excluding the two subjects with current major depressive disorder, CRF is still negatively correlated with PBI Care $(r=-384, n=35, p=0.02)$. Even after excluding the 19 male PD subjects previously reported on and the two subjects with current MDD, CSF CRF level was still inversely correlated with PBI Care score $(r=-0.659, p=0.003, n=16)$.

To examine categorical predictors of CSF CRF, CRF level was compared in the PD group divided by median split of PBI Care (median $=19$ ) into two equally sized groups: a high PBI PD $(n=18)$ and low PBI PD $(n=18)$, and normal controls $(n=17)$. One-way ANOVA, with the dependent variable of CSF CRF level compared across three groups, resulted in a significant overall model $(\mathrm{F}(2,50)=6.770$, $p=0.002)$, with a large effect size $\left(\eta^{2}=0.213\right)$. Follow-up tests were conducted to evaluate pairwise differences among the means, corrected for multiple comparisons and unequal variances using the Dunnet's C test. Low PBI PD subjects had significantly higher CSF CRF level than high PBI PD and normal control subjects. High PBI PD and normal control subjects did not significantly differ with respect to CSF CRF (Figures 1 and 2). Exploration of the effect of DSM-IV personality disorder on CSF CRF level was limited to paranoid $(n=8)$ and borderline $(n=6)$ personality disorder, due to the small number of other personality disorders in the sample. Compared to normal controls,

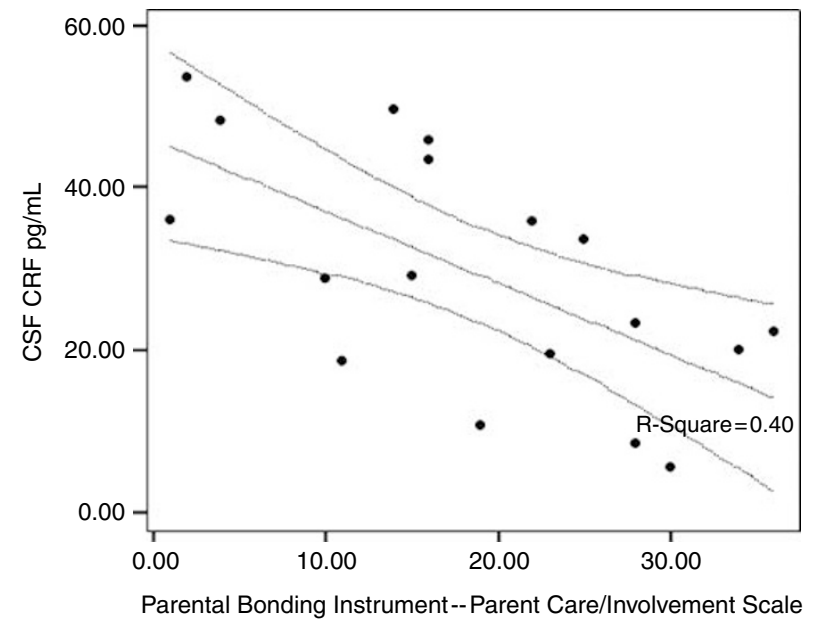

Figure I Scatterplot of PBI Care $\times$ CSF CRF level $(\mathrm{pg} / \mathrm{ml})$ in 18 PD subjects, excluding 19 subjects previously reported on in Lee et al, 2005 (Pearson's $r=-0.635, p=0.005, n=18$ ).

Table 3 Results of Linear Regression Analysis of Model Predicting CSF CRF (pg/ml) $(n=54)$

\begin{tabular}{|c|c|c|c|c|c|c|c|c|c|}
\hline Predictor & $\Delta R^{2}$ & $\mathbf{F}$ & $d, f$ & $p$ & B & SE & $\beta$ & $t$ & $P$ \\
\hline Step I & 0.057 & 1.532 & 2,51 & 0.23 & & & & & \\
\hline Sex & & & & & 0.054 & 5.809 & -0.034 & -0.256 & 0.799 \\
\hline Age & & & & & 0.522 & 0.306 & 0.232 & 1.706 & 0.094 \\
\hline Step 2 & 0.122 & 2.666 & 4,49 & 0.04 & & & & & \\
\hline Step 3 & 0.057 & 2.967 & 5,48 & 0.02 & & & & & \\
\hline $\mathrm{PBI} \times \mathrm{PD}$ & & & & & -13.020 & 6.857 & -0.651 & -1.899 & 0.06 \\
\hline
\end{tabular}




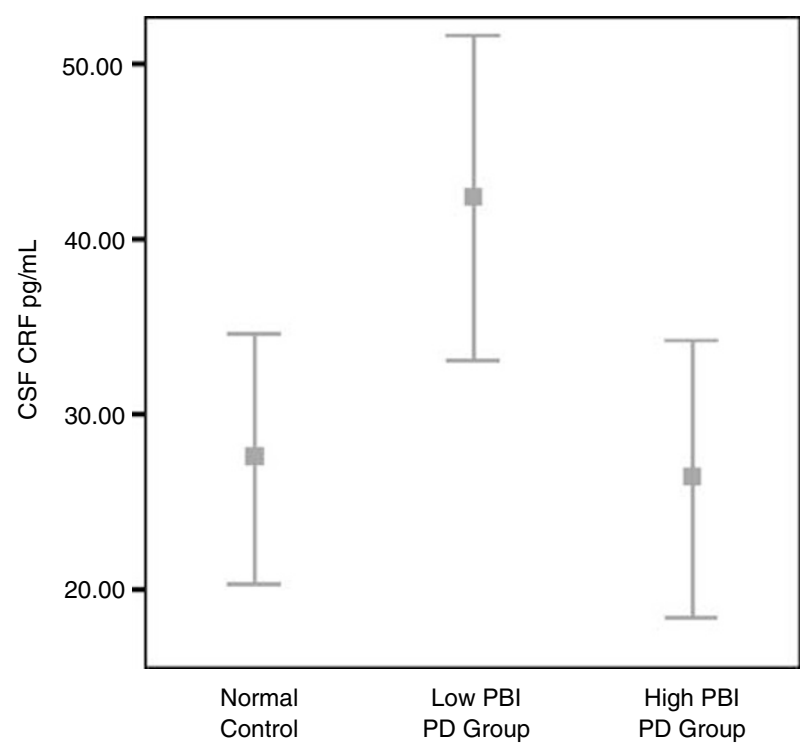

Figure 2 Mean CSF CRF $(\mathrm{pg} / \mathrm{ml})$ and $95 \%$ confidence interval of the mean in normal controls $(n=17)$, Low PBI care PD Subjects $(n=18)$, and high PBI care PD subjects $(n=18)$. Mean CSF CRF $(\mathrm{pg} / \mathrm{ml})$ in the low PBI PD group (44.I48, $S D=18.169$ ) was significantly higher than mean CSF CRF $(\mathrm{pg} / \mathrm{ml})$ in the normal control $(27.587, \mathrm{SD}=13.936)$ and high PBI PD group (26.366, SD $=15.90 \mathrm{I})$. No significant difference in mean CSF CRF was found between the normal control and high PBI PD group.

paranoid $\mathrm{PD}$, but not borderline $\mathrm{PD}$, was associated with elevated CSF CRF level (paranoid PD $=39.8 \pm 12.2$; normal controls $=27.1 \pm 13.2 \mathrm{pg} / \mathrm{ml} ; \mathrm{t}(25)=-2.313, p=0.03)$.

\section{DISCUSSION}

Consistent with the central hypothesis, after accounting for gender and age, CSF CRF was inversely related to PBI Care, indicating that higher CSF CRF was found in subjects reporting less parental care and involvement. The results were not due to the presence of current major depression, post-traumatic stress disorder, medication use, smoking history, height, or weight. The presence of PD does not determine CSF CRF level, but the test for a statistical interaction between PD and PBI Care on CSF CRF level was marginally significant, with PBI Care and CSF CRF inversely related in $\mathrm{PD}$, but not normal control subjects. This is accounted for by the significant restriction of range in PBI Care scores to the higher end in normal, non-PD samples; a larger sample would be necessary to adequately address the relationship between parental care and adult CSF CRF levels in normal controls. Of note, a previous investigation did find a relationship between CSF CRF and a different measure of early life stress in healthy volunteers not screened for PD (Carpenter et al, 2004).

If PD does not determine CSF CRF level, what is the clinical relevance of CSF CRF in PD? Our secondary, categorical hypothesis was that low parental care would separate out a subgroup of PD subjects with abnormally high CSF CRF levels. Accordingly, PD subjects with lower than median PBI Care score had higher CSF CRF levels than PD subjects with higher than median PBI Care score and normal control subjects. Interestingly, PD subjects reporting higher levels of parental care and involvement did not differ from normal control subjects with respect to CSF CRF level, indicating again that PD itself does not predict a high CSF CRF concentration. Exploratory analyses revealed that paranoid PD subjects, in relation to normal controls, had elevated CSF CRF levels. These results are of interest given the poor prognosis associated with paranoid $\mathrm{PD}$, but are highly preliminary and need replication.

The results replicate two previous reports of a positive relationship between history of childhood trauma (the conceptual opposite of parental care) and CSF concentrations of CRF. In a sample of adult depressives and normal controls subjects, early life stress in the preschool years predicted higher CSF CRF concentration, while preteen and perinatal early life stress was associated with decreased CSF CRF concentration. The finding was not driven by the presence of depression (Carpenter et al, 2004). We recently reported an analysis of male PD subjects who completed the childhood trauma questionnaire (CTQ). The CTQ is a retrospective measurement of perceived childhood trauma that is highly negatively correlated with PBI parental care score. Perceived history of childhood emotional neglect was positively correlated with CSF CRF (Lee et al, 2005). Analysis of the univariate correlation between CSF CRF and PBI parental care in the current sample minus the original 19 male PD subjects found a highly significant negative correlation of even greater magnitude. Thus, the relationship found in the present study is not being 'driven' by the subgroup of subjects in the previously published study.

The principal limitation of the study is retrospective assessment of parental care. Although some evidence supports the validity of retrospective self-assessments of childhood abuse and/or neglect (Widom and Morris, 1997; Widom et al, 1996), recall bias, which may be affected by the presence of PD or perhaps by stress hormone levels, cannot be ruled out as a confounding factor (Widom et al, 1999). Additionally, the cross-sectional nature of the study does not establish the temporal sequence of altered parental care and CSF CRF increases. A prospective design could establish the temporal sequence of altered parental care and CSF CRF level changes, but still would not be able to establish causality, and would be premature in young children given the risk of lumbar puncture. Clear ethical imperatives would forbid a randomized, prospective experiment measuring the effect of altered parental care on CSF CRF level. However, information is available from animal models of the effect of altered early life environment on central CRF function, in which experimental design can test causality. Rodent studies find that early life maternal infant interactions have a modulating effect on CSF CRF concentration (Plotsky and Meaney, 1993). Non-human primate studies utilizing the maternal stressor of the variable foraging paradigm have found that adult offspring of mothers exposed to this experimental paradigm have persistently elevated CSF CRF levels (Coplan et al, 1996, 1998), increased separation distress, and decreased affiliation with con-specifics (Andrews and Rosenblum, 1994). The mechanism through which altered early life parental care modulates adult CRF function may include decreased negative feedback at the level of hippocampus (Weaver 
et al, 2004), decreased hippocampal size (Vythilingam et al, 2002), altered inhibitory and excitatory inputs from frontallimbic circuits to the CRF neurons (Caldji et al, 2004), and altered serotonergic activity (Smythe et al, 1994; Mitchell et al, 1990).

The behavioral effect of elevated central CRF in humans is uncertain, but current understanding of the role of CRF in emotional processes indicates that CRF could play an important role in personality psychopathology. Acute stress causes upregulation of CRF in amygdala (Stout et al, 2002). Stimulation of amygdalar CRF receptors, and consequent increase in amygdalar metabolic activity, results in increased anxiety-like and decreased pro-social behaviors (Strome et al, 2002; DeVries et al, 2002). CRF is also released in the frontal cortex, where it signals the salience of environmental cues (Merali et al, 2004). Consistent with the prominent role of CRF in emotional processes, psychopathology such as depression, anxiety disorders, and suicide are associated with central CRF abnormalities (Nemeroff et al, 1984; Bissette et al, 1985; Bánki et al, 1987; Roy et al, 1987; Baker et al, 1999; Arató et al, 1986). Increased CRF is not elevated in all psychopathology, however, arguing against CRF being a nonspecific sign of psychopathology (Geracioti et al, 1997; Pitts et al, 1995). It is uncertain to what degree CSF CRF is a state or trait measure. Both CSF $\mathrm{CRF}$ and ACTH have been found to increase following the stress of lumbar puncture (Geracioti et al, 1997; Chappell et al, 1996). However, some evidence suggests that CSF CRF concentration measured acutely by lumbar puncture does reflect chronic CSF concentration (Geracioti et al, 1997; Bremner et al, 2003). Although it could also be argued that PD subjects, who score higher on measures of stress reactivity such as neuroticism, are more sensitive than other subjects to the psychological stress of lumbar puncture. Mean CRF concentration in the PD group $(39.9 \pm 27.8 \mathrm{pg} / \mathrm{ml})$ was comparable to those reported previously in clinical populations with depression (Carpenter et al, 2004), anxiety disorder (Fossey et al, 1996), acute alcohol withdrawal (Adinoff et al, 1996), schizophrenia (Nishino et al, 1998), in PTSD with and without psychosis (Sautter et al, 2003), and as measured by serial sampling in PTSD (Baker et al, 1999). It is of interest that CRF values in our normal controls $(27.1 \pm 13.2 \mathrm{pg} / \mathrm{ml})$ are comparable to those reported by Carpenter et al (2004) $(24.9 \pm 8.6 \mathrm{pg} / \mathrm{ml})$.

If replicated, the results of this study indicate that a subgroup of PD individuals who report decreased parental care and involvement in childhood have abnormally high central CRF levels. The magnitude of CRF difference is comparable to that found in previous studies of Axis I mood and anxiety disorders such as PTSD and MDD. One interpretation of the results is that decreased exposure to parental care results in elevated central CRF drive, possibly in interaction with unmeasured genetic factors. Altered CRF drive may in turn contribute to the development of personality disorder symptoms (ie paranoid anxiety). Given that history of early life trauma may be associated with nonresponse to antidepressant medications (Nemeroff, 2004), and that effective medical antidepressant treatment decreases CRF levels (Heuser et al, 1998), the findings from this study may offer important clues regarding the biology of difficult-to-treat psychopathology. Future studies are needed to investigate the moderating role of CRF-related genetic variability, and the behavioral consequences of both elevated and decreased brain CRF activity.

\section{ACKNOWLEDGEMENTS}

Editorial assistance was provided by Aaron TenHarmsel, Research Assistant. This project was supported in part by NIMH Grants 1RO3 MH066888-01 (Dr Lee) and RO1MH46948 and KO2MH00951 (Dr Coccaro). There are no conflicts of interest with regard to this work.

\section{REFERENCES}

Adinoff B, Anton R, Linnoila M, Guiodotti A, Nemeroff CB, Bissette $G$ (1996). Cerebrospinal fluid concentrations of corticotropin-releasing hormone (CRF) and diazepam-binding inhibitor (DBI) during alcohol withdrawal and abstinence. Neuropsychopharmacology 15: 288-295.

Andrews MW, Rosenblum LA (1994). The development of affiliative and agonistic social patterns in differentially reared monkeys. Child Dev 65: 1398-1404.

Arató M, Bánki CM, Nemeroff CB, Bissette G (1986). Hypothalamic pituitary adrenal axis and suicide. In: Mann JJ, Stanley M (eds). Psychobiology of Suicidal Behavior. New York Academy of Science: New York. pp 263-270.

Baker DG, West SA, Nicholson WE, Ekhator NN, Kasckow JW, Hill KK et al (1999). Serial CSF corticotropin-releasing hormone levels and adrenocortical activity in combat veterans with posttraumatic stress disorder. Am J Psychiatry 156: 585-588.

Bánki CM, Bissette G, Arató M, O’Connor L, Nemeroff CB (1987). CSF corticotropin-releasing factor-like immunoreactivity in depression and schizophrenia. Am J Psychiatry 144: 873-877.

Bernstein DP, Fink L (1988). Childhood Trauma Questionnaire. A Retrospective Self-Report: Manual. Psychological Corporation and Harcourt Brace: San Antonio, TX.

Bissette G, Spielman F, Stanley M, Bánki CM, Fink M, Stanley B et al (1985). Further studies of corticotropin releasing factor-like immunoreactivity in CSF of patients with affective disorders. Soc Neurosci Abstr 11: 133.

Bremner JD, Vythilingam M, Anderson G, Vermetten E, McGlashan T, Heninger $G$ et al (2003). Assessment of the hypothalamic-pituitary-adrenal axis over a 24-h diurnal period and in response to neuroendocrine challenges in women with and without childhood sexual abuse and posttraumatic stress disorder. Biol Psychiatry 54: 710-718.

Brierer LM, Yehuda R, Schmeidler J, Mitropoulou V, New AS, Silverman JM et al (2003). Abuse and neglect in childhood: relationship to PD diagnoses. CNS Spectrums 10: 737-740.

Caldji C, Diorio J, Anisman H, Meaney MJ (2004). Maternal behavior regulates benzodiazepine/GABA $\mathrm{A}$ receptor subunit expression in brain regions associated with fear in $\mathrm{BALB} / \mathrm{c}$ and C57BL/6 mice. Neuropsychopharmacology 29: 1344-1352.

Carpenter LL, Tyrka AR, McDougle CJ, Malison RT, Owens MJ, Nemeroff CB et al (2004). Cerebrospinal fluid corticotropinreleasing factor and perceived early life stress in depressed patients and healthy control subjects. Neuropsychopharmacology 29: 777-784.

Chappell P, Leckman J, Goodman W, Bissette G, Pauls D, Anderson $G$ et al (1996). Elevated cerebrospinal fluid corticotropin-releasing factor in Tourette's syndrome: comparison to obsessive compulsive disorder and normal controls. Biol Psychiatry 39: 776-783.

Coccaro EF, Kavoussi RJ, Sheline YI (1996). Impulsive aggression in PD: correlates with ${ }^{3} \mathrm{H}$-Paroxetine binding in the platelet. Archives of General Psychiatry 53: 531-536. 
Coplan JD, Andrews MW, Rosenblum LA, Owens MJ, Friedman S, Gorman JM et al (1996). Persistent elevations of cerebrospinal fluid concentrations of cotrioctropin releasing factor in adult nonhuman primates exposed to early-life stressors: implications for the pathophysiology of mood and anxiety disorders. Proc Natl Acad Sci USA 93: 1619-1623.

Coplan JD, Trost RC, Owens MJ, Cooper TB, Gorman JM, Nemeroff CB et al (1998). Cerebrospinal fluid concentrations of somatostatin and biogenic amines in grown primates reared by mothers exposed to manipulated foraging conditions. Arch Gen Psychiatry 55: 473-477.

Cutler NR, Hodes JE (1983). Assessing the noradrenergic system in normal aging: a review of methodology. Exp Aging Res 9: 123-127.

DeVries AC, Guptaa T, Cardillo S, Cho M, Carter CS (2002). Corticotropin-releasing factor induces social preferences in male prarie voles. Psychoneuroendocrinology 27: 705-714.

Fossey MD, Lydiard RB, Ballenger JC, Laraia MT, Bissette G, Nemeroff CB (1996). Cerebrospinal fluid corticotropin-releasing factor concentrations in patients with anxiety disorders and normal comparison subjects. Biol Psychiatry 39: 703-707.

Garno JL, Goldberg JF, Ramirez PM, Ritzler BA (2005). Impact of childhood abuse on the clinical course of bipolar disorder. $\mathrm{Br} J$ Psychiatry 186: 357.

Geracioti Jr TD, Loosen PT, Orth DN (1997). Low cerebrospinal fluid corticotropin-releasing hormone concentrations in eucortisolemic depression. Biol Psychiatry 42: 165-174.

Goodman M, New A, Siever L (2004). Trauma, genes, and the neurobiology of PDs. Ann NY Acad Sci 1032: 104-116.

Heuser I, Bissette G, Dettling M, Schweiger U, Gotthardt U, Schmider J et al (1998). Cerebrospinal fluid concentrations of corticotropin-releasing hormone, vasopressin, and somatostatin in depressed patients and healthy controls: response to amitriptyline treatment. Depress Anxiety 8: 71-79.

Johnson JG, Cohen P, Brown J, Smailes EM, Bernstein DP (1999). Childhood maltreatment increases risk for personality disorders during early adulthood. Arch Gen Psychiatry 56: 600-606.

Kasckow JW, Hagan M, Mulchahey JJ, Baker DG, Ekhator NN, Strawn JR et al (2001). The effect of feeding on cerebrospinal fluid corticotropin-releasing hormone levels in humans. Brain Res 904: 218-224.

Lee RJ, Kasckow JW, Geracioti T, Coccaro EF (2005). Childhood trauma and PD: positive correlation with adult CSF corticotropin-releasing factor concentrations. Am J Psychiatry 5: 995-997.

Merali Z, McIntosh J, anisman H (2004). Anticipatory cues differentially provoke in vivo peptidergic and monoaminergic release at the medial prefrontal cortex. Neuropsychopharmacology 29: 1409-1418.

Mitchell JB, Iny LJ, Meaney MJ (1990). The role of serotonin in the development and environmental regulation of Type II corticosteroid receptor binding in rat hippocampus. Dev Brain Res 55: 231-235.

Nemeroff CB (2004). Early-life adversity, CRF dysregulation, and vulnerability to mood and anxiety disorders. Psychopharmacol Bull 38: 14-20.

Nemeroff CB, Widerlov E, Bissette G, Walleus H, Karlsson I, Eklund $\mathrm{K}$ et al (1984). Elevated concentrations of CSF corticotropin-releasing factor-like immunoreactivity in depressed patients. Science 226: 1342-1344.
Nishino S, Mignot E, Benson KL, Zarcone VP (1998). Cerebrospinal fluid prostaglandins and corticotropin releasing factor in schizophrenics and controls: relationship to sleep architecture. Psychiatry Res 78: 141-150.

Parker G, Tupling H, Brown LB (1979). A Parental Bonding Instrument. Br J Med Psychol 52: 1-10.

Pfohl B, Blum N, Zimmerman M (1989). Structured Interview for the Diagnosis of DSM PDs. U. Iowa College of Medicine: Iowa City, IA.

Pitts AF, Samuelson SD, Meller WH, Bissette G, Nemeroff CB, Kathol RG (1995). Cerebrospinal fluid corticotropin-releasing hormone, vasopressin, and oxytocin concentrations in treated patients with major depression and controls. Biol Psychiatry 38: 330-335.

Plotsky PM, Meaney MJ (1993). Early, postnatal experience alters hypothalamic corticotropin-releasing factor (CRF) mRNA, median eminence CRF content and stress-induced release in adult rats. Brain Res Mol Brain Res 18: 195-200.

Roy A, Pickar D, Paul S, Doran A, Chrousos GP, Gold PW (1987). CSF corticotropin-releasing hormone in depressed patients and normal control subjects. Am J Psychiatry 144: 641-645.

Sautter FJ, Bissette G, Wiley J, Manguno-Mire G, Schoenbachler B, Myers L et al (2003). Corticotropin-releasing factor in posttraumatic stress disorder (PTSD) with secondary psychotic symptoms, nonpsychotic PTSD, and healthy control subjects. Biol Psychiatry 54: 1382-1388.

Smythe JW, Rowe WB, Meaney MJ (1994). Neonatal handling alters serotonin (5-HT) turnover and 5-HT-sub-2-receptor handling in selected brain regions: relationship to the handling effect of glucocorticoid receptor expression. Brain Res Dev Brain Res 80: 183-189.

Spitzer RL, Endicott J, Robins E (1978). Research diagnostic criteria: rationale and reliability. Arch Gen Psychiatry 35: 773-782.

Stout SC, Owens MJ, Nemeroff (2002). Regulation of corticotropin releasing factor neuronal systems and hypothalamic-pituitaryadrenal axis activity by stress and chronic antidepressant treatment. J Pharmacol Exp Ther 300: 1085-1092.

Strome EM, Wheler GHT, Higley JD, Loriaux DL, Suorni SJ, Doudet DJ (2002). Intracerebroventricular corticotropin-releasing factor increases limbic glucose metabolism and has social context-dependent behavioral effects in nonhuman primates. Proc Natl Acad Sci 99: 15749-15754.

Vythilingam M, Heim C, Newport J, Miller AH, Anderson E (2002). Childhood trauma associated with smaller hippocampal volume in women with major depression. Am J Psychiatry 159: 20722080.

Weaver IC, Diorio J, Sekl JR, Szyf M, Meaney MJ (2004). Early environmental regulation of hippocampal glucocorticoid receptor gene expression: characterization of intracellular mediators and potential genomic target sites. Ann NY Acad Sci 1024: $182-212$.

Widom CS, Morris S (1997). Accuracy of adult recollections of childhood victimization: Part 2. Childhood sexual abuse. Psychological Assessment 9: 34-46.

Widom CS, Spatz C, Shepart RL (1996). Accuracy of adult recollections of childhood victimization: Part 1. Childhood physical abuse. Psychol Assess 8: 412-421.

Widom CS, Weiler BL, Cottler LB (1999). Childhood victimization and drug abuse: a comparison of prospective and retrospective findings. J Consult Clin Psychol 67: 867-880. 